\title{
THEORETICAL ULTRAVIOLET SPECTRA OF B TYPE STARS : UNCERTAINTIES IN $\mathrm{G}$ VALUES
}

\section{Marijke Burger}

Astrophysical Institute, Vrije Universiteit, Brussel

Theoretical spectra have been computed (in LTE) for B type stars, in the wavelength range 2000-3000 $\AA$. The results show a good agreement with observations (S59 and Copernicus).

It will be shown that the most important factor that influences the resulting computed spectrum - at a resolution of $1.8 \mathrm{~A}^{0}$ or lower - is the completeness of the list of spectral lines used in the computations, owing to the large (number) density of lines in the ultraviolet.

At a resolution of $1.8 \AA$ a large error is tolerated on gf values, while identification of observed line features from the theoretical spectra remains possible.

The accuracy of the gf values becomes increasingly more important if one goes to higher spectral resolution, as will be shown by comparison of theoretical spectra with observations.

Our present knowledge of oscillator strengths is sufficient for identification of a large number of spectral lines in high resolution spectra; for a detailed analysis more accurate data are preferable in many cases. 